\title{
A web based dynamic MANA Nomogram for predicting the malignant cerebral edema in patients with large hemispheric infarction
}

Wenzhe Sun ${ }^{1}$, Guo Li ${ }^{1}$, Yang Song ${ }^{2}$, Zhou Zhu' ${ }^{1}$ Zhaoxia Yang ${ }^{3}$, Yuxi Chen ${ }^{4}$, Jinfeng Miao ${ }^{1}$, Xiaoyan Song ${ }^{1}$, Yan Lan ${ }^{1}$, Xiuli Qiu', Suiqiang Zhu ${ }^{1 *}$ and Yebin Fan ${ }^{5^{*}}$

\begin{abstract}
Background: For large hemispheric infarction (LHI), malignant cerebral edema (MCE) is a life-threatening complication with a mortality rate approaching $80 \%$. Establishing a convenient prediction model of MCE after LHI is vital for the rapid identification of high-risk patients as well as for a better understanding of the potential mechanism underlying MCE.

Methods: One hundred forty-two consecutive patients with LHI within $24 \mathrm{~h}$ of onset between January 1, 2016 and August 31, 2019 were retrospectively reviewed. MCE was defined as patient death or received decompressive hemicraniectomy $(\mathrm{DHC})$ with obvious mass effect ( $\geq 5 \mathrm{~mm}$ midline shift or Basal cistern effacement). Binary logistic regression was performed to identify independent predictors of MCE. Independent prognostic factors were incorporated to build a dynamic nomogram for MCE prediction.

Results: After adjusting for confounders, four independent factors were identified, including previously known atrial fibrillation (KAF), midline shift (MLS), National Institutes of Health Stroke Scale (NIHSS) and anterior cerebral artery (ACA) territory involvement. To facilitate the nomogram use for clinicians, we used the "Dynnom" package to build a dynamic MANA (acronym for MLS, ACA territory involvement, NIHSS and KAF) nomogram on web (http://www.MANA-nom. com) to calculate the exact probability of developing MCE. The MANA nomogram's C-statistic was up to $0.887 \pm 0.041$ and the AUC-ROC value in this cohort was 0.887 (95\%Cl, $0.828 \sim 0.934)$.

Conclusions: Independent MCE predictors included KAF, MLS, NIHSS, and ACA territory involvement. The dynamic MANA nomogram is a convenient, practical and effective clinical decision-making tool for predicting MCE after LHI in Chinese patients.
\end{abstract}

Keywords: Ischemic stroke, Large hemispheric infarction, Brain edema, Nomogram, Atrial fibrillation

\footnotetext{
*Correspondence: zhusuiqiang@163.com; hustfyb@hust.edu.cn

1 Department of Neurology, Tongji Hospital, Tongji Medical College,

Huazhong University of Science and Technology, No.1095 Jiefang Avenue,

Wuhan 430030, China

${ }^{5}$ School of Computer Science and Technology, Huazhong University of Science and Technology, No.1095 Jiefang Avenue, Wuhan 430030, China

Full list of author information is available at the end of the article
}

(C) The Author(s). 2020 Open Access This article is licensed under a Creative Commons Attribution 4.0 International License, which permits use, sharing, adaptation, distribution and reproduction in any medium or format, as long as you give appropriate credit to the original author(s) and the source, provide a link to the Creative Commons licence, and indicate if changes were made. The images or other third party material in this article are included in the article's Creative Commons licence, unless indicated otherwise in a credit line to the material. If material is not included in the article's Creative Commons licence and your intended use is not permitted by statutory regulation or exceeds the permitted use, you will need to obtain permission directly from the copyright holder. To view a copy of this licence, visit http://creativecommons.org/licenses/by/4.0/ The Creative Commons Public Domain Dedication waiver (http://creativecommons.org/publicdomain/zero/1.0/) applies to the data made available in this article, unless otherwise stated in a credit line to the data. 


\section{Background}

Large hemispheric infarction (LHI) is a severe ischemic stroke involving a large portion of MCA territory with significant morbidity and mortality [1]. For LHI, malignant cerebral edema (MCE) is a life-threatening complication with a mortality rate approaching $80 \%[2,3]$. MCE is characterized by a malignant course of rapid neurological deterioration associated with massive cerebral swelling between the second and fifth day after stroke onset $[4,5]$, subsequent increased intracranial pressure (ICP), midline shift and brain herniation. To date, effective conservative treatment of MCE remains unsolved [6]. Moreover, treatment for MCE had largely focused on symptomatic patients rather than on edema prevention. Given the exceptionally high mortality rate associated with MCE, understanding the underlying mechanisms and predictors in patients with MCE, thereby enabling the identification of patients who will benefit from early intervention and providing effective approaches for preventing MCE, are important.

Previous clinical research has indicated demographic, clinical and radiographic predictors for MCE [7-10]. Reported predictors of MCE included younger age, higher National Institutes of Health Stroke Scale (NIHSS) [7], larger parenchymal hypoattenuation on computed tomography (CT) [11], hyperdense artery sign and higher blood glucose [10]. In recent years, diffusion weighted imaging (DWI) has been extensively used for MCE prediction [7, 9]. However, these indicators are not precise enough, not easy to obtain, or their predictive role in clinical settings is limited by their hysteresis [12]. Meanwhile, most known predictors are described in the Western context. Although China has experienced rapid health transitions over the last four decades, the lifetime risk of stroke in China is significantly higher than the global average [13]. With stroke being the first cause of death in China [14]. Hence, MCE in Chinese patients is worth exploring.

Furthermore, traditional MCE scoring models enrolled NHISS and MLS as categorical variables for its convenience by clinical use, which may end up degrading precision [15-18]. Nomograms can get rid of that limitation but are not as clinically accessible as scoring models. Motivated by the above facts, we sought to establish a webbased nomogram, with balanced precision and practicability, to forecast MCE in Chinese patients with LHI.

\section{Methods}

\section{Patient selection}

This study continuously enrolled 157 adult patients diagnosed with LHI and admitted within $24 \mathrm{~h}$ to the neuro-intensive care unit (NICU) of Tongji Hospital at Huazhong University of Science and Technology between January 2016 and August 2019. This retrospective study was approved by our Institutional Review Board and the need for written informed consent was waived. LHI was defined as infarction involving $\geq 50 \%$ of the territory of the middle cerebral artery (MCA) in computed tomography (CT) scan or DWI infarct volume $>82 \mathrm{~mL}$ within $24 \mathrm{~h}$ of onset [19]. Infarct volume was calculated using the $\mathrm{ABC} / 2$ formula [20]. MCE was defined as patient death or received DHC with obvious mass effect on follow-up imaging examination ( $\geq 5 \mathrm{~mm}$ midline shift or Basal cistern effacement on CT or DWI). Patients were selected for $\mathrm{DHC}$ at our institution based on the criteria outlined in previously published trials [21]. All patients completed the baseline CT scan or DWI at admission and had no primary intracranial hemorrhage. Other inclusion criteria for our study were: 1) Chinese ethnicity; 2) age $\geq 18$ years; 3 ) first or recurrent acute stroke occurring within $24 \mathrm{~h}$ before admission. The exclusion criteria included: 1) Patients with terminal illness such as tumor, severe trauma, or other life-threatening diseases before admission; 2) patients without follow-up imaging examinations (CT scan or DWI) $24 \mathrm{~h}$ after onset; 3) death with secondary intracranial hemorrhage, acute myocardial infarction (AMI) or severe infection during hospitalization.

\section{Data collection}

Admission characteristics were recorded for all patients, consisting of age, gender, smoking and drinking history, preexisting hypertension, diabetes mellitus, previous stroke, preexisting coronary heart disease, admission temperature, admission systolic pressure and admission diastolic pressure and previous atrial fibrillation (AF). AF was identified as previously known AF (KAF), differentiating from AF detected after stroke (AFDAS) [22]. Recorded treatments included intravenous thrombolysis or endovascular intervention. Laboratory tests on admission included baseline fasting blood glucose (FBG) and HbA1c. All 142 patients underwent CT/DWI within 24 $\mathrm{h}$ of onset and the imaging data were evaluated by two experienced clinicians, blinded to the patients' outcome. Midline shift (MLS) was defined as the distance from the septum pellucida to the anatomic line anchored by the falx cerebri to the skull. Stroke severity was measured using the National Institutes of Health stroke scale (NIHSS). Cases without recorded NIHSS $(N=127$, $89.4 \%$ ) were not excluded from analysis. The NIHSS scores of all patients in this study were assessed by two experienced raters according to the patient's admission documented neurologic exams. All raters were blinded to the patients' outcome. An intra-rater reliability test was performed in 50 subjects, and the kappa values for MLS and NIHSS are 0.88 and 0.80 , respectively. 


\section{Statistical analysis}

Statistical analyses were performed using IBM SPSS Statistics 22.0 (SPSS Inc., Chicago, IL, United States) and $\mathrm{R}$ version 3.5.2 (Institute for Statistics and Mathematics, Vienna, Austria; http://www.r-project.org/). Statistical analyses to identify risk factors were performed using SPSS version 22.0 (Statistical Package for the Social Sciences) for Windows (SPSS, Chicago, IL). Continuous variables (including MLS) are expressed as the median and interquartile range (IQR) while categorical variables as percentages. We performed binary logistic regression to determine predictors independently associated with MCE. Univariate analyses were conducted using univariate logistic regression analysis. Variables with $P<0.05$ from the results of the univariate analyses were considered potential confounders and included in the multivariable model. The multivariable logistic regression using a backward stepwise method with input of variables if $p$-value $<0.05$ and backward elimination if $p$-value $>$
0.05. All $P$-values were two-sided, $P<0.05$ was considered statistically significant. Collinearity of variables that entered the multivariate logistic regression analysis was assessed by variation inflation factors $(<5$ being considered nonsignificant) and tolerance $(>0.2$ being considered nonsignificant).

The "rms" package and "Dynnom" package (cran.rproject. Org/web/packages/ rms) were used to construct a dynamic nomogram model. The performance of the dynamic nomogram was measured by the concordance index (C-index) and the area under the curve (AUC). The C-index was assessed by comparing nomogrampredicted probability versus observed probability. Bootstraps with 1000 resamples were applied to these activities. A higher C-index indicating better ability to separate patients with different MCE risk. Calibration curves were used to compare the predicted probability with the observed probability in the study. If the model calibration is correct, dots on the calibration plot should be close to a $45^{\circ}$ diagonal line.

Table 1 Demographic and clinical characteristics of patients with and without MCE

\begin{tabular}{|c|c|c|c|}
\hline Parameter & Non-ME $(N=101)$ & ME $(N=41)$ & $P$ \\
\hline Age, y, (IQR) & $58(51 \sim 67)$ & $64(57 \sim 71)$ & $0.005^{*}$ \\
\hline Gender, male, N (\%) & $70(69.3)$ & $26(63.4)$ & 0.497 \\
\hline Smoke, N (\%) & $53(52.5)$ & $21(51.2)$ & 0.892 \\
\hline Drink, N (\%) & 44 (43.6) & $14(34.1)$ & 0.302 \\
\hline Hypertension, N (\%) & $50(49.5)$ & $21(51.2)$ & 0.853 \\
\hline Diabetes mellitus, N (\%) & $18(17.8)$ & $9(22)$ & 0.570 \\
\hline Previous stroke, N (\%) & $16(15.8)$ & $13(31.7)$ & $0.037^{*}$ \\
\hline Preexisting coronary heart disease, N (\%) & $9(8.9)$ & $10(24.4)$ & $0.018^{*}$ \\
\hline KAF & $8(7.9)$ & $13(31.7)$ & $0.001^{*}$ \\
\hline \multicolumn{4}{|l|}{ Treatment, N (\%) } \\
\hline Conservative (reference) & 79 (78.2) & $34(82.9)$ & \\
\hline Intravenous thrombolysis & $16(15.8)$ & $4(9.8)$ & 0.362 \\
\hline Endovascular intervention & $6(5.9)$ & $3(7.3)$ & 0.839 \\
\hline NIHSS (IQR) & $18(15 \sim 20)$ & $21(20 \sim 22)$ & $<0.001^{*}$ \\
\hline MLS, mm (IQR) & $1.9(3.6 \sim 5.1)$ & $5.6(4.9 \sim 8.1)$ & $<0.001^{*}$ \\
\hline Baseline temperature, ${ }^{\circ} \mathrm{C}(\mathrm{IQR})$ & $36.5(36.4 \sim 36.8)$ & $36.5(36.5 \sim 37.1)$ & 0.170 \\
\hline ACA territory involvement, $\mathrm{N}(\%)$ & $9(8.9)$ & $18(43.9)$ & $<0.001^{*}$ \\
\hline PCA territory involvement, N (\%) & $27(26.7)$ & $24(58.5)$ & $<0.001^{*}$ \\
\hline Basal ganglia involvement, N (\%) & $60(59.4)$ & $37(90.2)$ & $0.001^{*}$ \\
\hline Cerebral hemisphere, right, N (\%) & $56(55.4)$ & $15(36.6)$ & $0.044^{*}$ \\
\hline Systolic pressure, mmHg, (IQR) & $145(127 \sim 166)$ & $141(120 \sim 159)$ & 0.168 \\
\hline Diastolic pressure, $\mathrm{mmHg}$, (IQR) & $83(73 \sim 95)$ & $80(74 \sim 92)$ & 0.871 \\
\hline $\mathrm{FBG}, \mathrm{mmol} / \mathrm{L},(\mathrm{IQR})$ & $5.9(5.4 \sim 6.8)$ & $7.1(5.8 \sim 9.2)$ & $0.002^{*}$ \\
\hline $\mathrm{HbA1c}$ \%, (IQR) & $5.6(5.3 \sim 6.1)$ & $5.7(5.3 \sim 6.1)$ & 0.378 \\
\hline
\end{tabular}

MCE: Malignant cerebral edema, IQR Interquartile range, KAF Previously known atrial fibrillation, NIHSS National Institutes of Health stroke scale, MLS Midline shift, ACA Anterior cerebral artery, PCA Posterior cerebral artery, FBG Baseline fasting blood glucose, HbA1c Glycosylated hemoglobin

${ }^{*} p<0.05$ in univariate analysis were included in multivariable logistic regression models for adjustment 


\section{Results}

\section{Patient characteristics}

In total, 142 patients with LHI were consecutively recruited in this study. Of all these potential subjects, 41 (28.9\%) finally developed MCE (39 died, 2 received DHC). As shown in Table 1, patients with MCE were older (median, 64 vs. 58 years; mean, 65 vs. 58; $p=$ $0.005)$, more likely to have preexisting coronary heart disease (24.4 vs. $8.9 \% ; p=0.018)$, previous stroke $(31.7$ vs. $15.8 \% ; p=0.037)$ or $\operatorname{KAF}(31.7$ vs. $7.9 \% ; p=0.001)$, higher baseline NIHSS (median, 21 vs. 18; mean, 21 vs. $17 ; p<0.001$ ), higher baseline fasting blood glucose (median, 7.1 vs. $5.9 \mathrm{mmol} / \mathrm{L}$; mean, 7.9 vs. $6.4 \mathrm{mmol} / \mathrm{L} ; p=$ 0.002 ) and greater admission MLS (median, 5.6 vs. 1.9 $\mathrm{mm}$; mean, 6.2 vs. $3.7 \mathrm{~mm} ; p<0.001)$. In addition, patients with left hemisphere infarction (63.4 vs. 44.6\%; $p=0.044$ ) and patients with concurrent ACA (43.9 vs. $8.9 \%$; $p<0.001)$, PCA (58.5 vs. $26.7 \% ; p<0.001)$ territory or basal ganglia ( 90.2 vs. $59.4 \% ; p=0.001$ ) infarction were more likely to develop into MCE.

\section{Independent predictors and dynamic nomogram for predicting MCE}

Variables showing $p<0.05$ in univariate logistic regression were included in multivariable logistic regression models for adjustment (Table 1). No significant statistical collinearity was observed for these variables. After adjusting by potential confounders, $\mathrm{KAF}(\mathrm{aOR}=4.68$, 95\% CI, $1.42 \sim 15.42)$, MLS (aOR = 1.30, 95\% CI, 1.04 1.62), NIHSS ( $\mathrm{aOR}=1.33,95 \% \mathrm{CI}, 1.07 \sim 1.66)$ and $\mathrm{ACA}$ territory involvement $(\mathrm{aOR}=4.64,95 \% \mathrm{CI}, 1.59 \sim 13.60)$ were independent predictors (Table 2).

All independent MCE predictors (Table 2) were considered to construct a nomogram (Fig. 1). This nomogram can predict MCE individually according to the various patient conditions. Each of the four independent predictors were projected upward to the value of the "Points" on top to get a score, with a point range from 0 to 100. Points assigned to the corresponding factors were summed to calculate the "total points". The total score was then converted into an individual MCE risk. The higher the total score, the higher the risk of MCE. The predictive accuracy of the nomogram was validated

Table 2 Multivariate logistic regression model for MCE

\begin{tabular}{lllll}
\hline Parameter & $\boldsymbol{\beta}$ & SE & $\boldsymbol{P}$ & OR $(95 \% \mathrm{Cl})$ \\
\hline KAF & 1.54 & 0.61 & $0.011^{*}$ & $4.68(1.42 \sim 15.42)$ \\
MLS & 0.26 & 0.11 & $0.023^{*}$ & $1.30(1.04 \sim 1.62)$ \\
NIHSS & 0.28 & 0.11 & $0.012^{*}$ & $1.33(1.07 \sim 1.66)$ \\
ACA & 1.54 & 0.55 & $0.005^{*}$ & $4.64(1.59 \sim 13.60)$
\end{tabular}

MCE Malignant cerebral edema, KAF Previously known atrial fibrillation, NIHSS National Institutes of Health stroke scale, MLS Midline shift, ACA Anterior cerebral arter, OR Odds Ratio, $\mathrm{Cl}$ Confidence Interval, SE Standard Error *Statistically significant at $p<0.05$ level, two-sided using 1000 bootstrap samples, with a Harrell's c-index value of $0.887 \pm 0.041$ (Fig. 2). The model was also internally validated in this cohort with an AUC-ROC value of 0.887 (95\%CI, $0.828 \sim 0.934)$. Furthermore, to facilitate the use of the nomogram for clinicians, we used the "Dynnom" package to build an operation interface on a web page (www.MANAnom.com) to calculate the exact probability of developing MCE (Fig. 3). To help others to understand the MANA nomogram, we input four different combinations of the independent predictors, correspond to four imaginary patients. In Fig. 3, the four lines in different colors in part B corresponds to the predictions and $95 \% \mathrm{CI}$ of these four patients. Part C shows these four patients' actual numerical values of predictions and $95 \% \mathrm{CI}$.

\section{Discussions}

We identified four independent MCE predictors in this study, Three of which consistent with previous studies, including NIHSS, MLS and ACA territory involvement $[17,18]$. However the association between KAF and MCE is still controversial. On this basis we developed a visual MANA (MLS, ACA territory involvement and KAF) nomogram to assess the risk of MCE development of MCE in Chinese patients with LHI. The c-statistic of the MANA nomogram was up to $0.887 \pm 0.041$ while the AUC-ROC in this cohort was 0.887 (95\%CI, 0.828 0.934).

For the past few years, with the development of cardiac monitoring technologies, physicians have noticed atrial fibrillation (AF) after ischemic stroke or transient ischemic attack (TIA). About $23.7 \%$ patients without AF before stroke later develop AF [23], termed AF detected after stroke (AFDAS). Nevertheless, the relationship between KAF and MCE has never been clarified. Currently, KAF is considered as the cardiogenic AF, which was mainly caused by cardiac remodeling, while AF detected after stroke (AFDAS) may be composed of multiple AF types, including preexisting but newly diagnosed atrial fibrillation (cardiogenic AF) and newly emerged atrial fibrillation (neurogenic AF) [24]. Neurogenic AF is the main type of AFDAS, which may be caused by the inflammatory response and dysfunction of the autonomic regulation of the cardiac rhythm [24, 25]. Based on this mechanistic difference between KAF and AFDAS, the effect of AFDAS on stroke severity may also vary from that of KAF. Previous research found that stroke patients with KAF have a higher rate of death or stroke recurrence (including hemorrhagic and ischemic stroke) than patients with AFDAS, but the difference was unadjusted [25]. To verify this supposition, we set up another multivariable regression to assess the differences in MCE risk among the sinus rhythm (SR), AFDAS and KAF (Table 3). After adjusting for confounders, we found that 


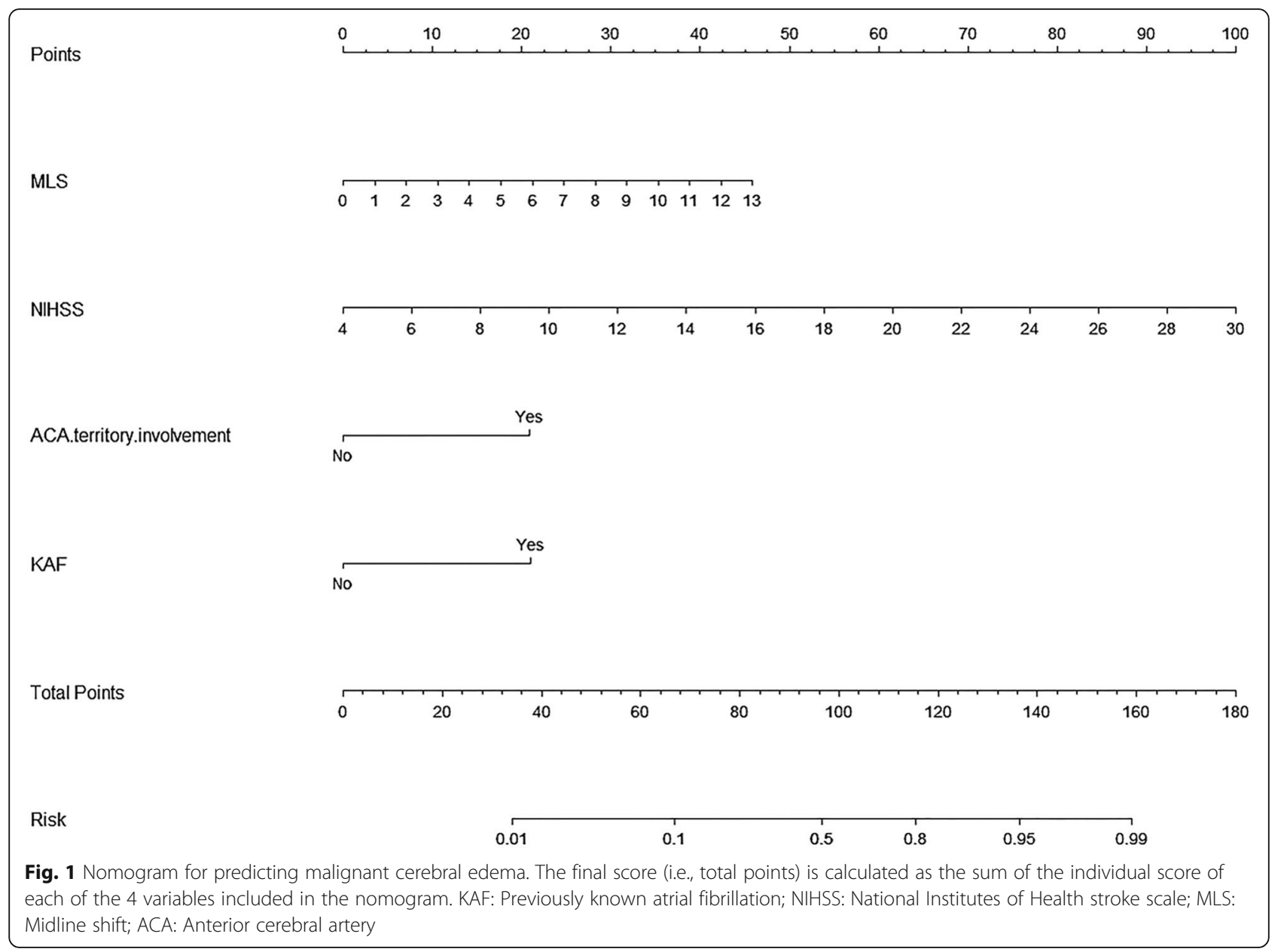
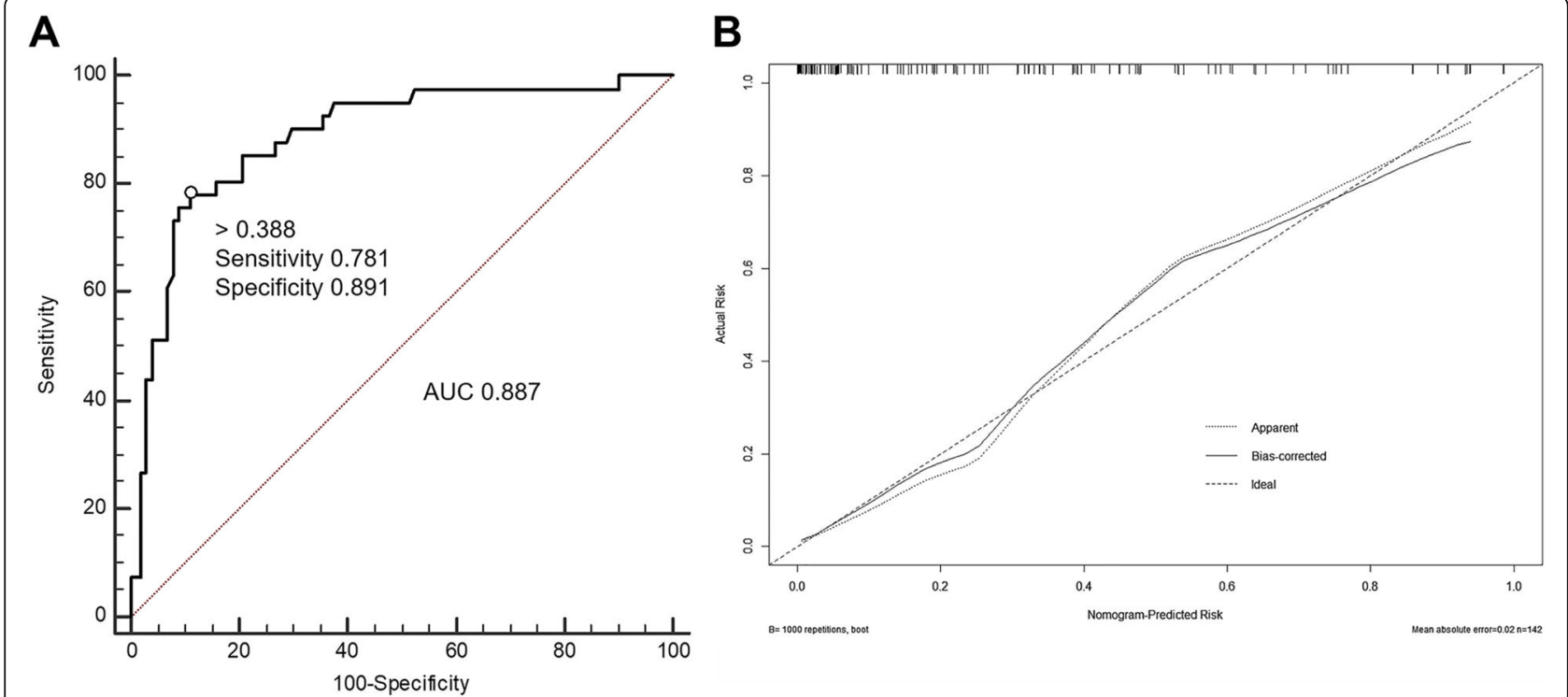

Fig. 2 a: ROC curve of the nomogram used for predicting malignant cerebral edema in Chinese patients; $\mathbf{b}$ : Calibration curves for the nomogram used for predicting malignant cerebral edema. Dashed line is reference line where an ideal nomogram would lie. Dotted line is the performance of nomogram, while the solid line corrects for any bias in nomogram. AUC: Area under curve. ROC: receiver operating characteristic 


\section{Web Based Dynamic MANA Nomogram for Predicting the Malignant Cerebral Edema}

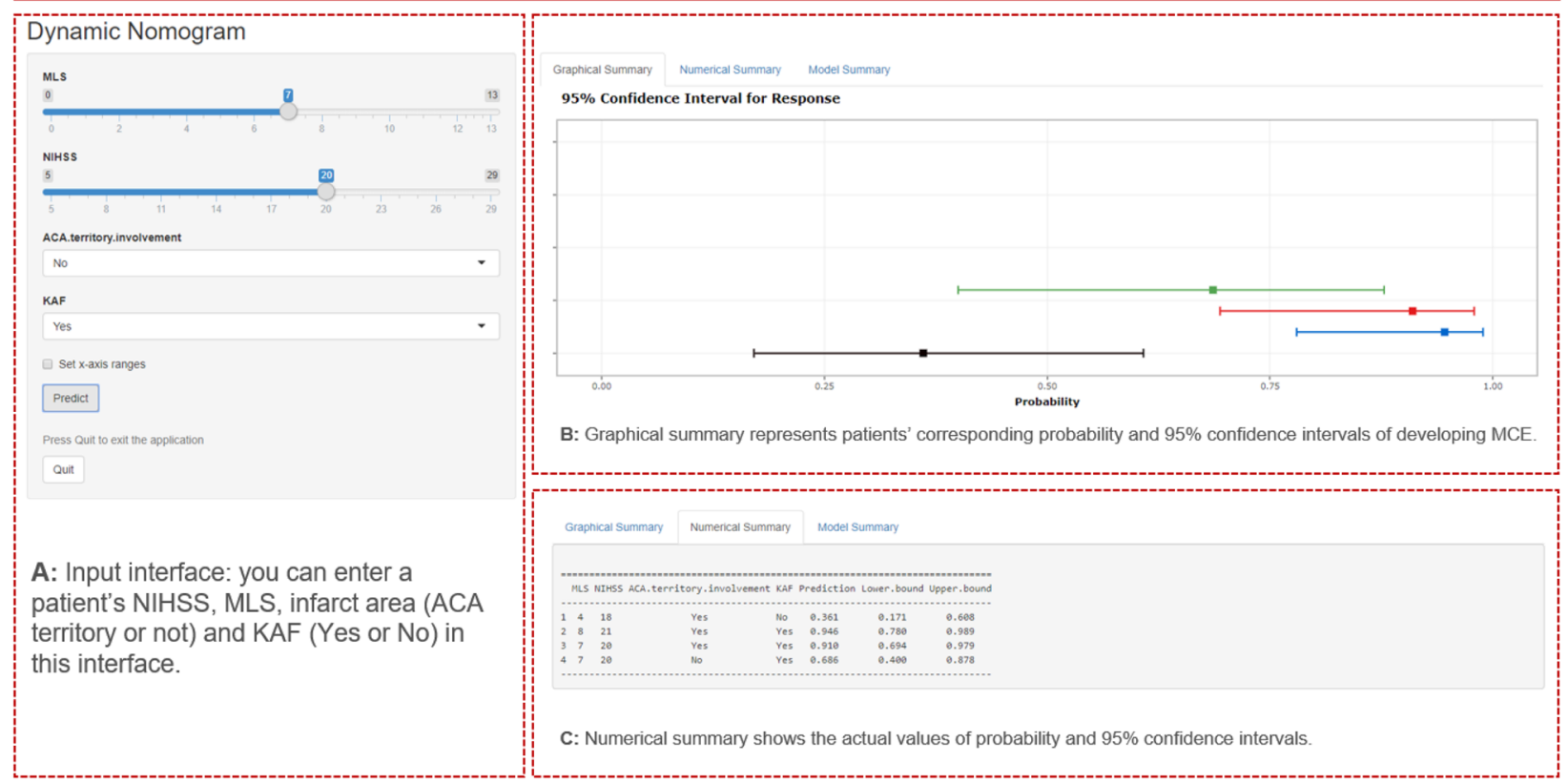

Fig. 3 Operation interface of nomogram on web page. After entering a patient's NIHSS, MLS, infarct area (ACA territory or not) and KAF (Yes or No) on http://www.MANA-nom.com, the neurologist can get the patient's corresponding probability of developing MCE. a: Input interface, you can enter a patient's NIHSS, MLS, infarct area (ACA territory or not) and KAF (Yes or No) in this interface. b: Graphical summary represents patients' corresponding probability and 95\% confidence intervals of developing MCE. c: Numerical summary shows the actual values of probability and 95\% confidence intervals. MLS: Midline shift; NIHSS: National Institutes of Health stroke scale; ACA: Anterior cerebral artery; KAF: Previously known atrial fibrillation

compared to patients with SR or AFDAS, patients with KAF had significantly higher risk of MCE (adjusted OR 4.29, 95\% CI, $1.28 \sim 14.36$ ). However, the risk between SR and AFDAS did not significantly differ. One possible reason is that patients with KAF had more severe

Table 3 Comparison of the risk of MCE among SR, AFDAS and KAF

\begin{tabular}{lllll}
\hline Parameter & $\boldsymbol{\beta}$ & SE & $\boldsymbol{P}$ & OR $(\mathbf{9 5 \% C l})$ \\
\hline $\begin{array}{c}\text { Rhythm } \\
\text { SR (reference) }\end{array}$ & & & & \\
$\quad$ AFDAS & -0.69 & 0.87 & 0.428 & $0.50(0.09 \sim 2.77)$ \\
KAF & 0.46 & 0.62 & $0.018^{*}$ & $4.29(1.28 \sim 14.36)$ \\
MLS & 0.25 & 0.11 & $0.026^{*}$ & $1.29(1.03 \sim 1.61)$ \\
NIHSS & 0.28 & 0.11 & $0.011^{*}$ & $1.33(1.07 \sim 1.66)$ \\
ACA & 1.63 & 0.57 & $0.004^{*}$ & $5.11(1.68 \sim 15.54)$ \\
\hline
\end{tabular}

MCE Malignant cerebral edema, SR Sinus rhythm, AFDAS Atrial fibrillation detected after stroke, KAF Previously known atrial fibrillation, NIHSS National Institutes of Health stroke scale, MLS Midline shift, ACA Anterior cerebral artery, OR Odds Ratio, $C I$ Confidence Interval, SE Standard Error

*Statistically significant at $p<0.05$ level, two-sided hypoperfusion, leading to greater infarct growth and larger infarcts [26]. Our research also suggests that patients with KAF may have more severe stroke than patients with AFDAS (mean NIHSS 20 vs 18; mean infarct volume $231.1 \mathrm{~mL}$ vs $191.5 \mathrm{~mL}$ ). Additionally, it is noticeable that risk factors of cardiac remodeling, such as endothelin-1 and matrix metalloproteinase, are also associated with brain edema [27-30]. Furthermore, neurogenic AF as "functional AF", may have less AF burden than cardiogenic AF, which can also influence patient prognosis [31]. It is also worth noting that only $2.7 \%$ patients with AF in China received anticoagulant treatment [32], which may also be associated with the more severe ischemic stroke and brain edema incidence in China.

For patients with LHI, ACA territory involvement often hints the existence of larger infarction or more proximal vascular occlusion, such as carotid $\mathrm{T}$ or internal carotid artery (ICA) occlusion, less hemispheric collateral flow and greater volume of edematous brain tissue [15]. Further, the NIHSS score is correlated with stroke severity and infarct volume [10,33], and MLS is a visual indicator on $\mathrm{CT}$ or MRI images, even sonographic 
monitoring, for assessing the severity of brain edema [34, 35]. Previous studies included NHISS and MLS as categorical variables into scoring models of malignant brain edema for the convenience of clinical use [10,18]. However, compared to the continuous use of NIHSS and MLS, the categorical use loses precision. Despite the nomogram being able to circumvent that limitation, it has lesser practicability than scoring models. To make up for these deficiencies, we established a web operation interface (http://www.MANA-nom.com) for the MANA nomogram, which combines practicality and accuracy. Additionally, we did not collect data from CT angiography (CTA), DWI or special measurement techniques [17, 36-38], considering that the model needed to be available and propagable.

Unlike previous nomograms that roughly calculate an approximation, the dynamic MANA nomogram can provide an exact value. Furthermore, it's convenient for neurologists all over the world. After entering a patient's NIHSS, MLS, infarct area (ACA territory or not) and KAF (Yes or No) on http://www.MANA-nom.com, the neurologist can get the patient's corresponding probability of developing MCE. The MANA nomogram can also be used to identify patients who need early surgical treatment, or aid in the decision-making process for patients with high likelihood of MCE of LHI.

\section{Limitations}

This study has several limitations. First, since the data of this study was retrospectively collected from a single center in China, some information may not be accurate enough. Most patients didn't have recorded NIHSS, and the calculated NHISS may have discrepancy with the actual situation. Second, distinguishing KAF and AFDAS through history and electrocardiograph (ECG) during hospitalization may not be sufficiently rigorous. Moreover, limited by current ECG monitoring technology, quite a few paroxysmal AF was undetected, which underestimated the number of patients with AFDAS. Third, defining the primary outcome as death with brain edema or received DHC might have made us neglect the fact that some patients developed severe brain edema but pulled through without DHC at discharge. In addition, this network prediction model sometimes leads to system crashes, which can be resolved by clicking "Quit" and re-logging in. Despite these limitations, our study provided a widely available prediction model for neurologists to assess the risk of MCE in patients with LHI.

\section{Conclusions}

Our study aimed to finding the risk factors of MCE and establishing a convenient and accurate risk model to forecast MCE for Chinese patients with LHI. We found that KAF may increase the risk of MCE in patients with
LHI. The dynamic MANA nomogram can help neurologists make clinical decisions and discuss prognosis to patients' families. Additionally, further external validation through prospective, multi-center, large-scale trials of this model are also necessary.

\begin{abstract}
Abbreviations
LHI: Large Hemispheric Infarction; MCE: Malignant cerebral edema; DHC: Decompressive hemicraniectomy; KAF: Previously known atrial fibrillation; MLS: Midline shift; NIHSS: National Institutes of Health Stroke Scale; ACA: Anterior cerebral artery; ICP: Intracranial pressure; CT: Computed tomography; DWI: Diffusion weighted image; NICU: Neuro-intensive care unit; MCA: Middle cerebral artery; IQR: Interquartile range; PCA: Posterior cerebral artery; FBG: Fasting blood glucose; HbA1c: Glycosylated hemoglobin; AF: Atrial fibrillation; AFDAS: AF detected after stroke; FBG: Fasting blood glucose; ICC: Intraclass correlation efficient; C-index: Concordance index; AUC: Area under the curve value; OR: Odds Ratio; Cl: Confidence Interval; SE: Standard Error; TIA: Transient ischemic attack; ICA: Internal carotid artery; CTA: CT angiography
\end{abstract}

\section{Acknowledgements}

Not applicable.

\section{Authors' contributions}

WS: writing-original draft. GL, YS, JM, YL, XQ: collected, analyzed and interpreted the data. ZY, WS: analyzed all imaging data. YF, ZZ, SZ, YC: contributed substantially to study design and revised the manuscript. The author(s) read and approved the final manuscript.

\section{Funding}

This work was financially supported by the National Key R\&D Program of China [grant number 2017YFC1310000], the Fundamental Research Funds for the Central Universities [grant number 2018KFYXMPT015] and Hubei Technological Innovation Special Fund (CN) [grant number 2019ACA132]. The funders had no role in study design, data collection and analysis, decision to publish, or preparation of the manuscript.

\section{Availability of data and materials}

The datasets used and analysed during the current study are available from the corresponding author on reasonable request.

\section{Ethics approval and consent to participate}

This retrospective study was approved by Review Board of Tongji Hospital at Huazhong University of Science and Technology and written informed consent was waived.

\section{Consent for publication}

Not applicable.

\section{Competing interests}

The authors report no conflict of interest concerning the materials or methods used in this study or the findings specified in this paper.

\section{Author details}

'Department of Neurology, Tongji Hospital, Tongji Medical College, Huazhong University of Science and Technology, No.1095 Jiefang Avenue, Wuhan 430030, China. ${ }^{2}$ School of Medicine and Health Management; Tongji Medical College, Huazhong University of Science and Technology, Wuhan 430030, China. ${ }^{3}$ Department of Radiology, Tongji Hospital, Tongji Medical College, Huazhong University of Science and Technology, No.1095 Jiefang Avenue, Wuhan 430030, China. ${ }^{4}$ The Solomon H. Snyder Department of Neuroscience, Johns Hopkins University School of Medicine, Baltimore, MD 21205, USA. ${ }^{5}$ School of Computer Science and Technology, Huazhong University of Science and Technology, No.1095 Jiefang Avenue, Wuhan 430030, China. 
Received: 24 May 2020 Accepted: 17 September 2020

Published online: 29 September 2020

\section{References}

1. Hacke W, Schwab S, Horn M, Spranger M, De Georgia M, von Kummer R 'Malignant' middle cerebral artery territory infarction: clinical course and prognostic signs. Arch Neurol. 1996:53(4):309-15.

2. Heinsius T, Bogousslavsky J, Van Melle G. Large infarcts in the middle cerebral artery territory. Etiology and outcome patterns. Neurology. 1998; 50(2):341-50.

3. Huttner HB, Schwab S. Malignant middle cerebral artery infarction: clinical characteristics, treatment strategies, and future perspectives. Lancet Neurol. 2009;8(10):949-58.

4. Frank JI. Large hemispheric infarction, deterioration, and intracranial pressure. Neurology. 1995;45(7):1286-90.

5. Shaw CM, Alvord EC Jr, Berry RG. Swelling of the brain following ischemic infarction with arterial occlusion. Arch Neurol. 1959;1:161-77.

6. Hofmeijer J, van der Worp HB, Kappelle LJ. Treatment of space-occupying cerebral infarction. Crit Care Med. 2003;31(2):617-25.

7. Thomalla G, Hartmann F, Juettler E, Singer OC, Lehnhardt FG, Kohrmann M, et al. Prediction of malignant middle cerebral artery infarction by magnetic resonance imaging within 6 hours of symptom onset: a prospective multicenter observational study. Ann Neurol. 2010;68(4):435-45.

8. Hofmeijer J, Algra A, Kappelle LJ, van der Worp HB. Predictors of lifethreatening brain edema in middle cerebral artery infarction. Cerebrovasc Dis. 2008;25(1-2):176-84.

9. Oppenheim C, Samson Y, Manai R, Lalam T, Vandamme X, Crozier S, et al. Prediction of malignant middle cerebral artery infarction by diffusionweighted imaging. Stroke. 2000;31(9):2175-81.

10. Thoren M, Azevedo E, Dawson J, Egido JA, Falcou A, Ford GA, et al. Predictors for cerebral edema in acute ischemic stroke treated with intravenous thrombolysis. Stroke. 2017;48(9):2464-71.

11. Wu S, Yuan R, Wang Y, Wei C, Zhang S, Yang X, et al. Early prediction of malignant brain edema after ischemic stroke. Stroke. 2018;49(12):2918-27.

12. Campbell JK, Houser OW, Stevens JC, Wahner HW, Baker HL Jr, Folger WN Computed tomography and radionuclide imaging in the evaluation of ischemic stroke. Radiology. 1978;126(3):695-702.

13. Feigin VL, Nguyen G, Cercy K, Johnson CO, Alam T, Parmar PG, et al. Global, regional, and country-specific lifetime risks of stroke, 1990 and 2016. N Engl J Med. 2018;379(25):2429-37.

14. Wu S, Wu B, Liu M, Chen Z, Wang W, Anderson CS, et al. Stroke in China: advances and challenges in epidemiology, prevention, and management. Lancet Neurol. 2019;18(4):394-405.

15. Kasner SE, Demchuk AM, Berrouschot J, Schmutzhard E, Harms L, Verro P, et al. Predictors of fatal brain edema in massive hemispheric ischemic stroke. Stroke. 2001;32(9):2117-23.

16. Jo K, Bajgur SS, Kim H, Choi HA, Huh PW, Lee K. A simple prediction score system for malignant brain edema progression in large hemispheric infarction. PLoS One. 2017;12(2):e0171425.

17. Shimoyama T, Kimura K, Uemura J, Yamashita S, Saji N, Shibazaki K, et al. The DASH score: a simple score to assess risk for development of malignant middle cerebral artery infarction. J Neurol Sci. 2014;338(1-2):102-6.

18. Ong CJ, Gluckstein J, Laurido-Soto O, Yan Y, Dhar R, Lee JM. Enhanced detection of edema in malignant anterior circulation stroke (EDEMA) score: a risk prediction tool. Stroke. 2017:48(7):1969-72.

19. Sheth KN, Elm J, Beslow LA, Sze GK, Kimberly WT. Glyburide advantage in malignant edema and stroke (GAMES-RP) trial: rationale and design. Neurocrit Care. 2016:24(1):132-9.

20. Sims JR, Gharai LR, Schaefer PW, Vangel M, Rosenthal ES, Lev MH, et al. $\mathrm{ABC} / 2$ for rapid clinical estimate of infarct, perfusion, and mismatch volumes. Neurology. 2009;72(24):2104-10.

21. Vahedi K, Hofmeijer J, Juettler E, Vicaut E, George B, Algra A, et al. Early decompressive surgery in malignant infarction of the middle cerebral artery: a pooled analysis of three randomised controlled trials. Lancet Neurol. 2007; 6(3):215-22.

22. Seiffge DJ, Tagawa M. Insights into atrial fibrillation newly diagnosed after stroke: can the brain rule the heart? Neurology. 2018;90(11):493-4.

23. Sposato LA, Cipriano LE, Saposnik G, Ruiz Vargas E, Riccio PM, Hachinski V. Diagnosis of atrial fibrillation after stroke and transient ischaemic attack: a systematic review and meta-analysis. Lancet Neurol. 2015;14(4):377-87.
24. Cerasuolo JO, Cipriano LE, Sposato LA. The complexity of atrial fibrillation newly diagnosed after ischemic stroke and transient ischemic attack: advances and uncertainties. Curr Opin Neurol. 2017;30(1):28-37.

25. Hsieh CY, Lee CH, Wu DP, Sung SF. Characteristics and outcomes of ischemic stroke in patients with known atrial fibrillation or atrial fibrillation diagnosed after stroke. Int J Cardiol. 2018;261:68-72.

26. Tu HT, Campbell BC, Christensen S, Desmond PM, De Silva DA, Parsons MW, et al. Worse stroke outcome in atrial fibrillation is explained by more severe hypoperfusion, infarct growth, and hemorrhagic transformation. Int J Stroke. 2015;10(4):534-40

27. Lo AC, Chen AY, Hung VK, Yaw LP, Fung MK, Ho MC, et al. Endothelin-1 overexpression leads to further water accumulation and brain edema after middle cerebral artery occlusion via aquaporin 4 expression in astrocytic end-feet. J Cereb Blood Flow Metab. 2005;25(8):998-1011.

28. Rosenberg GA. Matrix metalloproteinases in brain injury. J Neurotrauma. 1995;12(5):833-42.

29. Nakazawa Y, Ashihara T, Tsutamoto T, Ito M, Horie M. Endothelin-1 as a predictor of atrial fibrillation recurrence after pulmonary vein isolation. Heart Rhythm. 2009;6(6):725-30.

30. Anne W, Willems R, Roskams T, Sergeant P, Herijgers P, Holemans P, et al. Matrix metalloproteinases and atrial remodeling in patients with mitral valve disease and atrial fibrillation. Cardiovasc Res. 2005:67(4):655-66.

31. Go AS, Reynolds K, Yang J, Gupta N, Lenane J, Sung SH, et al. Association of Burden of atrial fibrillation with risk of ischemic stroke in adults with paroxysmal atrial fibrillation: the KP-RHYTHM study. JAMA Cardiol. 2018;3(7): 601-8.

32. Zhou Z, Hu D. An epidemiological study on the prevalence of atrial fibrillation in the Chinese population of mainland China. J Epidemiol. 2008; 18(5):209-16.

33. Woo D, Broderick JP, Kothari RU, Lu M, Brott T, Lyden PD, et al. Does the National Institutes of Health stroke scale favor left hemisphere strokes? NINDS t-PA stroke study group. Stroke. 1999;30(11):2355-9.

34. Gerriets T, Stolz E, Konig S, Babacan S, Fiss I, Jauss M, et al. Sonographic monitoring of midline shift in space-occupying stroke: an early outcome predictor. Stroke. 2001;32(2):442-7.

35. Gerriets T, Stolz E, Modrau B, Fiss I, Seidel G, Kaps M. Sonographic monitoring of midline shift in hemispheric infarctions. Neurology. 1999; 52(1):45-9.

36. Beck C, Kruetzelmann A, Forkert ND, Juettler E, Singer OC, Kohrmann M, et al. A simple brain atrophy measure improves the prediction of malignant middle cerebral artery infarction by acute DWI lesion volume. J Neurol. 2014:261(6):1097-103

37. Kauw F, Bennink E, de Jong H, Kappelle LJ, Horsch AD, Velthuis BK, et al. Intracranial Cerebrospinal Fluid Volume as a Predictor of Malignant Middle Cerebral Artery Infarction. Stroke. 2019;50(6):STROKEAHA119024882.

38. Kim H, Jin ST, Kim YW, Kim SR, Park IS, Jo KW. Predictors of malignant brain edema in middle cerebral artery infarction observed on CT angiography. J Clin Neurosci. 2015:22(3):554-60.

\section{Publisher's Note}

Springer Nature remains neutral with regard to jurisdictional claims in published maps and institutional affiliations.

Ready to submit your research? Choose BMC and benefit from:

- fast, convenient online submission

- thorough peer review by experienced researchers in your field

- rapid publication on acceptance

- support for research data, including large and complex data types

- gold Open Access which fosters wider collaboration and increased citations

- maximum visibility for your research: over $100 \mathrm{M}$ website views per year

At $\mathrm{BMC}$, research is always in progress.

Learn more biomedcentral.com/submission 